\title{
Characterization of Integrons and Quinolone Resistance in Clinical Escherichia coli Isolates in Mansoura City, Egypt
}

\author{
Shaymaa H. Abdel-Rhman $\mathbb{D}^{1,2}$ Rehab M. Elbargisy $\mathbb{D D}^{1,3}$ and Dina E. Rizk $\mathbb{D}^{1}$ \\ ${ }^{1}$ Microbiology and Immunology Department, Faculty of Pharmacy, Mansoura University, Mansoura, Egypt \\ ${ }^{2}$ Department of Pharmaceutics and Pharmaceutical Biotechnology, Faculty of Pharmacy, Taibah University, \\ AlMadinah Al Munawwarah, Saudi Arabia \\ ${ }^{3}$ Department of Pharmaceutics, College of Pharmacy, Jouf University, Sakaka, Al-Jouf, Saudi Arabia
}

Correspondence should be addressed to Shaymaa H. Abdel-Rhman; shaymaahassan@mans.edu.eg

Received 28 June 2021; Revised 12 August 2021; Accepted 20 August 2021; Published 6 September 2021

Academic Editor: Ahmed Majeed Al-Shammari

Copyright (c) 2021 Shaymaa H. Abdel-Rhman et al. This is an open access article distributed under the Creative Commons Attribution License, which permits unrestricted use, distribution, and reproduction in any medium, provided the original work is properly cited.

\begin{abstract}
Escherichia coli is a common pathogen in both humans and animals. Quinolones are used to treat infections caused by Gramnegative bacteria, but resistance genes emerged. Only scarce studies investigated the association between plasmid-mediated quinolone resistance (PMQR) genes and integrons in clinical isolates of E. coli. The current study investigated the prevalence of quinolone resistance and integrons among 134 clinical E. coli isolates. Eighty (59.70\%) isolates were quinolone-resistant, and 60/ $134(44.77 \%)$ isolates were integron positive with the predominance of class I integrons (98.33\%). There was a significant association between quinolone resistance and the presence of integrons $(P<0.0001)$. Isolates from Urology and Nephrology Center and Gastroenterology Hospital were significantly quinolone-resistant and integron positive $(P \leq 0.0005)$. Detection of PMQR genes on plasmids of integron-positive isolates showed that the active efflux pump genes oqxAB and qepA had the highest prevalence $(72.22 \%)$, followed by the aminoglycoside acetyltransferase gene (aac $\left.\left(6^{\prime}\right)-I b-c r, 66.67 \%\right)$ and the quinolone resistance genes $(q n r, 61.11 \%)$. Amplification and sequencing of integrons' variable regions illustrated that no quinolone resistance genes were detected, and the most predominant gene cassettes were for trimethoprim and aminoglycoside resistance including $d f r A 17$, $d f r B 4$, and $d f r A 17$-aadA5. In conclusion, this study reported the high prevalence of PMQR genes and integrons among clinical E. coli isolates. Although PMQR genes are not cassette-born, they were associated with integrons' presence, which contributes to the widespread of quinolone resistance in Egypt.
\end{abstract}

\section{Introduction}

Despite being a well-known member of the normal flora for both humans and animals [1], the pathogenic strains of Escherichia coli cause a wide variety of infections including the skin and soft tissue, urinary tract, gastrointestinal tract, and central nervous system [2]. The severity of infections can vary from mild to life-threatening conditions based on the virulence capacity and antimicrobial resistance of the bacterium.

Fluoroquinolones are broad-spectrum synthetic antibiotics that can successfully treat infections caused by Enterobacteriaceae. Unfortunately, resistance to fluoroquinolones is increasing that is mediated chromosomally by modifications in their targets such as DNA gyrase and topoisomerase
IV [3]. Plasmid-mediated quinolone resistance (PMQR) is now spreading among Gram-negative bacteria. Three main mechanisms are employed for PMQR resistance. The first is the modification of quinolones' targets by qnr proteins [encoded by quinolone resistance genes ( $q n r$ genes)]. Secondly, fluoroquinolones are modified through acetylation by the aminoglycoside acetyltransferase enzyme (encoded by the $a a c\left(6^{\prime}\right)-I b-c r$ gene). Lastly, excretion of hydrophobic fluoroquinolones by active efflux pumps such as QepA and OqxAB (encoded by qep, oqxA, and $o q x B$ genes) [4].

Integrons are genetic elements that account for the dissemination of antimicrobial resistance genes in different species of bacteria. They are found on plasmids or transposons, which facilitate their transfer between bacterial cells 
[5]. Two main parts constitute an integron. The first part is made up of an integrase gene (intI), an integration site (attI), and a promoter. The second part includes an attachment site (attC) for a gene cassette. This cassette has a single gene and mostly an open reading frame but no promoter. When attI and attC sites recombine, genes carried by gene cassettes can be expressed through the integron promoter [6]. Gene cassettes integrated into integrons carry antimicrobial resistance genes that facilitate dissemination of antimicrobial resistance upon transfer of plasmids or transposons that include the integrons $[7,8]$. Although different classes of integrons have been discussed before, class I and II integrons remain the most prevalent ones among resistant clinical isolates $[9,10]$.

Although the association between $\mathrm{PMQR}$ and integrons among environmental isolates has been reported in several studies [8, 11-13], only few studies investigated the association between them in clinical E. coli isolates, and the investigations of the location of these genes on the variable region gene cassettes were scarce. Therefore, our study aimed to investigate the prevalence and association of quinolone resistance and integrons among clinical E. coli isolates. Additionally, the prevalence of $\mathrm{PMQR}$ genes among integron-positive isolates was studied.

\section{Materials and Methods}

2.1. Bacterial Isolates. During the period from February to August 2019, a total of 134 nonreplicate clinical E. coli isolates were obtained from different clinical specimens [urine (72 isolates), throat swabs (2 isolates), stool (51 isolates), blood (1 isolate), and wound exudate (8 isolates)]. They were collected from 5 different hospitals located in Mansoura city, Egypt [Urology and Nephrology Center (UNC), Gastroenterology Hospital (GEH), Microbiology Diagnostic Infection Control Unit (MDICU), Specialized Medical Hospital (SMC), and Mansoura University Children Hospital (MUCH)]. Identification of bacterial isolates was done according to laboratory biochemical standard methods [14]. The study fulfills the ethical guidelines approved by "The Research Ethics Committee, Faculty of Pharmacy, Mansoura University" that follow the Code of Ethics of World Medical Association (Declaration of Helsinki involving the use and handling of human subjects; Code Number: 2021-9).

2.2. Determination of Quinolone Resistance. Resistance of the isolates to four quinolone antibiotics including ciprofloxacin $(10 \mu \mathrm{g})$, levofloxacin $(5 \mu \mathrm{g})$, norfloxacin $(10 \mu \mathrm{g})$, and ofloxacin $(5 \mu \mathrm{g})$ was determined using the standard Kirby-Bauer disk diffusion method following the CLSI guidelines [15].

2.3. Genomic and Plasmid DNA Extraction. Genomic DNA was obtained from all isolates using the modified boiling technique [16]. The filtrate was stored at $-20^{\circ} \mathrm{C}$ and used as a template for polymerase chain reaction (PCR) amplification of integrase genes.
The selection of isolates for plasmid DNA extraction was based on the results obtained from PCR amplification of integrons on total DNA in addition to quinolone sensitivity testing. Plasmid DNA extraction was done using the GeneJET Plasmid Miniprep kit (K0502, EU, Lithuania).

2.4. PCR Amplification of Integrase Genes, Gene Cassettes, and $(P M Q R)$ Genes. Multiplex PCR was used for the detection of class I, II, and III integrons in all isolates using primers intI1, intI2, and intI 3 , respectively (Table 1 ). The variable regions of class I and II integrons and PMQR genes were amplified by PCR on plasmids extracted from selected 43 isolates using the primers listed in Table 1. PCR reaction mixture and cycling conditions were performed as previously described.

2.5. Restriction Fragment Length Polymorphism (RFLP) and Characterization of Gene Cassettes. To investigate the similarity between the gene cassettes of the different isolates, RFLP of purified PCR products of variable regions of class I and II integrons (extracted from the agarose gel using GenJet Gel extraction kit (K0691, EU, Lithuania)) was detected after digestion by restriction enzyme HinfI (CutSmart, New England Biolabs) [21] and sequenced on an automated sequencer (ABI Prism 3100). The resulted sequences were assembled using the CodonCode Aligner (version 9.0.1). BLAST (http://blast.ncbi.nlm.nih.gov/Blast.cgi) against the GenBank database was performed for sequence comparison and annotation.

2.6. Nucleotide Sequence Accession Numbers. The integron gene cassette nucleotide sequences identified in this study have been recorded in the GenBank database (GenBank accessions no. MW770320 to MW770348).

2.7. Statistical Analysis. GraphPad InStat (Fisher's exact and Chi-square tests) was used to analyze the results statistically. Results with $P$-value $\leq 0.05$ were considered of statistical significance.

\section{Results}

3.1. Resistance to Quinolones. The results of the antimicrobial sensitivity test illustrated that resistance to tested quinolones was significantly prevalent among isolates. It was found that 80 (59.70\%), 79 (58.95\%), 78 (58.20\%), and 77 (57.46\%) of isolates were resistant to norfloxacin, ciprofloxacin, ofloxacin, and levofloxacin, respectively.

It was found that urine isolates were significantly resistant to the tested quinolones $(P<0.0001)$. Besides, these isolates were significantly associated with UNC $(P<0.0001)$ and GEH $(P=0.0005)$.

3.2. PCR Amplification of Integrase Genes, Gene Cassettes, and PMQR Genes. Detection of integrase genes on genomic DNA showed that 60 isolates $(44.8 \%)$ harbored the genes. Integron I was significantly prevalent (59 isolates, 98.33\%; $P<0.0001$ ), followed by integron II (5 isolates $(8.33 \%), 4$ of 
Table 1: Primer sequences and conditions used in PCR amplification.

\begin{tabular}{|c|c|c|c|c|c|}
\hline Target gene & & Sequence $5^{\prime}-3^{\prime}$ & Annealing temperature $\left({ }^{\circ} \mathrm{C}\right)$ & Product size (bp) & Reference \\
\hline$q n r A$ & $\begin{array}{l}\mathrm{F} \\
\mathrm{R}\end{array}$ & $\begin{array}{l}\text { AGAGGATTTCTCACGCCAGG } \\
\text { TGCCAGGCACAGATCTTGAC }\end{array}$ & 60 & 580 & {$[17]$} \\
\hline$q n r B$ & $\begin{array}{l}\mathrm{F} \\
\mathrm{R}\end{array}$ & $\begin{array}{l}\text { GGMATHGAAATTCGCCACTG } \\
\text { TTTGCYGYYCGCCAGTCGAA }\end{array}$ & 60 & 264 & {$[17]$} \\
\hline$q n r C$ & $\begin{array}{l}\mathrm{F} \\
\mathrm{R}\end{array}$ & $\begin{array}{c}\text { GGGTTGTACATTTATTGAATC } \\
\text { TCCACTTTACGAGGTTCT }\end{array}$ & 52 & 307 & {$[18]$} \\
\hline$q n r D$ & $\begin{array}{l}\mathrm{F} \\
\mathrm{R}\end{array}$ & $\begin{array}{c}\text { CGAGATCAATTTACGGGGAATA } \\
\text { AACAAGCTGAAGCGCCTG }\end{array}$ & 52 & 465 & {$[19]$} \\
\hline$q n r S$ & $\begin{array}{l}\mathrm{F} \\
\mathrm{R}\end{array}$ & $\begin{array}{l}\text { GCAAGTTCATTGAACAGGCT } \\
\text { TCTAAACCGTCGAGTTCGGCG }\end{array}$ & 60 & 428 & {$[17]$} \\
\hline$q e p A$ & $\begin{array}{l}\mathrm{F} \\
\mathrm{R}\end{array}$ & $\begin{array}{l}\text { CTGCAGGTACTGCGTCATG } \\
\text { CGTGTTGCTGGAGTTCTTC }\end{array}$ & 52 & 403 & {$[20]$} \\
\hline$o q x A$ & $\begin{array}{l}\mathrm{F} \\
\mathrm{R}\end{array}$ & $\begin{array}{l}\text { GACAGCGTCGCACAGAATG } \\
\text { GGAGACGAGGTTGGTATGGA }\end{array}$ & 57 & 339 & {$[20]$} \\
\hline$o q \times B$ & $\begin{array}{l}\mathrm{F} \\
\mathrm{R}\end{array}$ & $\begin{array}{l}\text { CGAAGAAAGACCTCCCTACCC } \\
\text { CGCCGCCAATGAGATACA }\end{array}$ & 57 & 240 & {$[20]$} \\
\hline$a a c\left(6^{\prime}\right)-I b$ & $\begin{array}{l}\mathrm{F} \\
\mathrm{R}\end{array}$ & $\begin{array}{c}\text { TTGCGATGCTCTATGAGTGGCTA } \\
\text { CTCGAATGCCTGGCGTGTTT }\end{array}$ & 60 & 482 & {$[18]$} \\
\hline IntI1 & $\begin{array}{l}\mathrm{F} \\
\mathrm{R}\end{array}$ & $\begin{array}{l}\text { GGTCAAGGATCTGGATTTCG } \\
\text { ACATGCGTGTAAATCATCGTC }\end{array}$ & 60 & 436 & {$[21]$} \\
\hline IntI2 & $\begin{array}{l}\mathrm{F} \\
\mathrm{R}\end{array}$ & $\begin{array}{l}\text { CACGGATATGCGACAAAAAGG } \\
\text { TGTAGCAAACGAGTGACGAAATG }\end{array}$ & 60 & 788 & {$[21]$} \\
\hline IntI3 & $\begin{array}{l}\mathrm{F} \\
\mathrm{R}\end{array}$ & $\begin{array}{c}\text { AGTGGGTGGCGAATGAGTG } \\
\text { TGTTCTTGTATCGGCAGGTG }\end{array}$ & 60 & 600 & {$[21]$} \\
\hline $\begin{array}{l}5^{\prime} C S \\
3^{\prime} C S \\
\end{array}$ & $\begin{array}{l}\mathrm{F} \\
\mathrm{R}\end{array}$ & $\begin{array}{l}\text { GGCATCCAAGCAGCAAG } \\
\text { AAGCAGACTTGACCTGA }\end{array}$ & 58 & Variable & {$[21]$} \\
\hline $\begin{array}{l}\text { attI2-F } \\
\text { orf } X-R\end{array}$ & $\begin{array}{l}\mathrm{F} \\
\mathrm{R}\end{array}$ & $\begin{array}{l}\text { GACGGCATGCACGATTTGTA } \\
\text { GATGCCATCGCAAGTACGAG }\end{array}$ & 58 & Variable & {$[21]$} \\
\hline
\end{tabular}

F: forward and R: reverse.

TABLE 2: The PMQR genes distribution among different sources.

\begin{tabular}{lccccccccc}
\hline Source (no. of isolates) & $q n r A$ & $q n r B$ & $q n r C$ & $q n r D$ & $q n r S$ & $o q x A$ & $o q x B$ & $q e p A$ & $a a c\left(6^{\prime}\right)-I b$ \\
\hline Urine (32) & 3 & 2 & - & 3 & $12^{* *}$ & 6 & $15^{* * *}$ & $12^{* * *}$ & $19^{* * *}$ \\
Stool (5) & - & - & - & - & 3 & 1 & 3 & 2 & 4 \\
Wound (3) & - & - & - & - & 2 & - & - & - & - \\
Throat swab (2) & - & - & - & - & 1 & - & - & - \\
Blood (1) & 1 & - & - & - & 1 & - & - & - \\
Total (43) & $4(9.30 \%)$ & $2(4.65 \%)$ & $0(0 \%)$ & $3(6.97 \%)$ & $19(44.19 \%)$ & $7(16.28 \%)$ & $20(46.51 \%)$ & $14(32.56 \%)$ & $24(55.81 \%)$ \\
\hline
\end{tabular}

${ }^{* *} P$-value $=0.004$ and ${ }^{* * *} P<0.0001$

them coexist with integrons I) and integron III [3 isolates (5\%) that have also integron I].

Generally, integrons were significantly detected in isolates from UNC and GEH $(P<0.0001)$. A significant association of quinolone resistance and integrons was observed $(P$ value $=0.0061)$, where $43 / 80(53.75 \%)$ of quinolone-resistant isolates harbored integrons genes. These 43 isolates were significantly associated with UNC and GEH $(P<0.0001)$. All urine isolates having integrons were also quinolone-resistant $(P<0.0001)$.

PMQR genes were detected on plasmid DNA of the 36/ 43 isolates $(83.72 \%)$ that showed phenotypic quinolone resistance and harbored integrons. The results in Table 2 showed that $a a c\left(6^{\prime}\right)-I b, o q x B, q n r S$, and $q e p A$ were the most prevalent genes. None of the test isolates carried the $q n r C$ gene. Seven isolates did not harbor any of the tested genes. The distribution of PMQR genes among different sources was illustrated in Table 2. It showed that all genes were significantly detected in urine samples $(P<0.0001)$, while isolates from a throat swab and blood carried one and two genes, respectively.

Figure 1 illustrates the obtained 23 gene profiles. Isolates that carried 5 genes were all isolated from urine samples from UNC. Nine profiles were shown by more than 1 isolate (Pr1-Pr4, Pr6, Pr8, Pr11, Pr12, and Pr19). The most predominant profile was $\operatorname{Pr} 4$ (5 isolates, 11.62\%). qnrA, qnrB, $q n r D$, and $o q x A$ genes were not detected as a single-gene profile. The $q n r B$ gene was associated with profiles that carried 5 genes. The genes coexisted with each other without any significant association. 


\begin{tabular}{|c|c|c|c|c|c|c|c|}
\hline $\begin{array}{l}\text { No. of } \\
\text { genes }\end{array}$ & No of isolates (\%) & Profile No. & Profile & & & & \\
\hline 1 gene & $\begin{array}{l}3(7.0) \\
2(4.7) \\
2(4.7) \\
5(11.6)\end{array}$ & $\begin{array}{l}1 \\
2 \\
3 \\
4\end{array}$ & $\begin{array}{c}q n r S \\
o q x B \\
q e b A \\
a a c\left(6^{\prime}\right)-I b\end{array}$ & & & & \\
\hline Total & $12(27.9)$ & & & & & & \\
\hline 2 genes & $\begin{array}{l}1(2.3) \\
2(4.7) \\
1(2.3) \\
2(4.7)\end{array}$ & $\begin{array}{l}5 \\
6 \\
7 \\
8\end{array}$ & $\begin{array}{l}q n r A \\
q n r S \\
q n r S \\
o q \times B\end{array}$ & $\begin{array}{c}q n r S \\
o q \times B \\
a a c\left(6^{\prime}\right)-I b \\
a a c\left(6^{\prime}\right)-I b\end{array}$ & & & \\
\hline Total & $6(13.9)$ & & & & & & \\
\hline 3 genes & $\begin{array}{l}1(2.3) \\
1(2.3) \\
2(4.7) \\
2(4.7)\end{array}$ & $\begin{array}{l}9 \\
10 \\
11 \\
12\end{array}$ & $\begin{array}{l}q n r S \\
q n r S \\
q n r S \\
o q \times B\end{array}$ & $\begin{array}{l}o q x A \\
q e b A \\
o q x B \\
q e b A\end{array}$ & $\begin{array}{c}a a c\left(6^{\prime}\right)-I b \\
a a c\left(6^{\prime}\right)-I b \\
q e b A \\
a a c\left(6^{\prime}\right)-I b\end{array}$ & & \\
\hline Total & $6(13.9)$ & & & & & & \\
\hline 4 genes & $\begin{array}{l}1(2.3) \\
1(2.3) \\
1(2.3) \\
1(2.3) \\
1(2.3) \\
1(2.3) \\
2(4.7) \\
1(2.3)\end{array}$ & $\begin{array}{l}13 \\
14 \\
15 \\
16 \\
17 \\
18 \\
19 \\
20\end{array}$ & $\begin{array}{l}q n r A \\
q n r A \\
q n r D \\
q n r D \\
q n r S \\
q n r S \\
q n r S \\
o q x A\end{array}$ & $\begin{array}{l}q n r S \\
o q x A \\
o q x A \\
o q x B \\
o q x A \\
o q x A \\
o q x B \\
o q x B\end{array}$ & $\begin{array}{c}o q \times B \\
o q \times B \\
o q \times B \\
q e b A \\
o q \times B \\
q e b A \\
q e b A \\
q e b A\end{array}$ & $\begin{array}{l}a a c\left(6^{\prime}\right)-I b \\
a a c\left(6^{\prime}\right)-I b \\
a a c\left(6^{\prime}\right)-I b \\
a a c\left(6^{\prime}\right)-I b \\
a a c\left(6^{\prime}\right)-I b \\
a a c\left(6^{\prime}\right)-I b \\
a a c\left(6^{\prime}\right)-I b \\
\text { aac }\left(6^{\prime}\right)-I b\end{array}$ & \\
\hline Total & $9(20.9)$ & & & & & & \\
\hline 5 genes & $\begin{array}{l}1(2.3) \\
1(2.3) \\
1(2.3)\end{array}$ & $\begin{array}{l}21 \\
22 \\
23\end{array}$ & $\begin{array}{l}q n r A \\
q n r B \\
q n r D\end{array}$ & $\begin{array}{l}q n r B \\
q n r S \\
q n r S\end{array}$ & $\begin{array}{l}q n r S \\
o q x B \\
o q x A\end{array}$ & $\begin{array}{l}o q x B \\
q e b A \\
q e b A\end{array}$ & $\begin{array}{l}a a c\left(6^{\prime}\right)-I b \\
a a c\left(6^{\prime}\right)-I b \\
a a c\left(6^{\prime}\right)-I b\end{array}$ \\
\hline Total & $3(7.0)$ & & & & & & \\
\hline Total & 36 isolates & & & & & & \\
\hline
\end{tabular}

FIgURE 1: The gene profile of PMQR genes among the 36 clinical E. coli isolates.

3.3. Integrons and Characterization of Gene Cassettes. The sizes of the integron gene cassettes and their distribution among tested isolates are shown in Table 3. The investigation of integron gene cassettes revealed that integron I variable regions were amplified in 31/42 isolates (73.81\%), where 27 isolates showed 1 variable region with sizes ranging from $350 \mathrm{bp}$ to $2,200 \mathrm{bp}$. Integron II variable regions with a size of $2,200 \mathrm{bp}$ were amplified in $2 / 4(50 \%)$ isolates. Variable 
regions of $1,600 \mathrm{bp}(P<0.0001)$ and $600 \mathrm{bp}$ were more prevalent among isolates.

The digestion of the amplified variable regions of integrons I and II with HinfI restriction enzyme resulted in seven different patterns (P1-P7) and two different patterns (P8 and P9), respectively. Figure 2 illustrates the restriction patterns corresponding to each size. The variable region of $1,600 \mathrm{bp}$ gave the pattern P4 and was found in eighteen isolates. P7 that represents a $600 \mathrm{bp}$ amplicon was found in eight isolates.

Gene sequencing of the amplified variable regions showed that seven different gene cassettes were detected among 23 class I integron-positive isolates and two gene cassettes in $2 / 4$ of class II integron-positive isolates. Five gene cassettes had one gene either of dihydrofolate reductase ( $d f r$ family) or aminoglycosides resistance (aad family). Three gene cassettes included both $d f r$ and aad genes.

The detected genes belonged to trimethoprim resistance ( $d f r A 1, d f r A 7, d f r A 12, d f r A 17$, and $d f r B 4)$, aminoglycosides resistance (aadA1, aadA2, aadA5, and $\operatorname{aadA22})$, and streptothricin resistance (sat2; streptothricin acetyltransferase). There was also an open reading frame of unknown function (orfF).

The most common gene cassette was $d$ frA17 $(n=9$, $31.03 \%)$, followed by $d f r B 4(n=8,27.58 \%), d f r A 17$-aadA5 $(n=5,17.24 \%)$, aadA2-orfF-dfrA12 $(n=2,6.89 \%)$, and aadA22 ( $n=2,6.89 \%)$. dfrA1-sat2-aadA1, dfrA7, and drfA 12 gene cassettes were represented by one isolate each $(3.44 \%)$. dfrA17-aadA5 gene cassette was detected in integrons I and II (Table 4).

Table 5 shows the characterization of the 43 E. coli isolates including the isolation source, gene profiles, type of integron, and gene cassettes. It illustrated that the isolate may have more than one variable region with different gene cassettes. Moreover, it showed that the variable regions of approximately $1,600 \mathrm{bp}$ were similar as they carry either ( $d f r A 17$-aadA5) or $d f r A 17$. Integron-I-positive isolates that carried the $d f r A 17$ gene cassette also harbored the aadA5 gene cassette that had a frameshift mutation by addition and deletion of several nucleotides, which resulted in several internal stop codons and gaps.

\section{Discussion}

Since the discovery of PMQR genes in 1998, a wide distribution of these determinants, especially in members of Enterobacteriaceae, was observed. This may be due to the broad use of fluoroquinolone antibiotics in human medicine, veterinary medicine, and the environment [22]. In the current study, fluoroquinolones' resistance was highly prevalent in the tested isolates (57.46-59.70\%). A similar result was reported by a study performed in Egypt [23]. In contrast, previous studies from several countries [24-28] reported that most of the tested isolates were susceptible to fluoroquinolones (70-90\%). The main causes behind antibiotic resistance are the misuse of antibiotics, improper prescription by physicians, and unawareness of patients who do not follow dosage regimens [29]. Furthermore, the use of quinolones in agriculture may account for an increase in
TABLE 3: The distribution of integron variable region's sizes among tested E. coli isolates.

\begin{tabular}{|c|c|c|c|}
\hline \multicolumn{2}{|l|}{ Band no. (B) } & Variable regions' size (bp) & $\begin{array}{c}\text { No. of isolates } \\
(\%)\end{array}$ \\
\hline \multirow{13}{*}{ Class I integron } & 1 & 2,200 & $1(2.38)$ \\
\hline & 2 & 1,800 & $2(4.76)$ \\
\hline & 3 & 1,600 & $15(35.71)$ \\
\hline & 4 & 1,000 & $1(2.38)$ \\
\hline & 5 & 850 & $1(2.38)$ \\
\hline & 6 & 750 & $1(2.38)$ \\
\hline & 7 & 600 & $5(11.90)$ \\
\hline & 8 & 350 & $1(2.38)$ \\
\hline & 9 & $1,000,750$ & $1(2.38)$ \\
\hline & 10 & $1,600,600$ & $2(4.76)$ \\
\hline & 11 & $1,600,850,600$ & $1(2.38)$ \\
\hline & 12 & Without PCR products & $11(26.19)$ \\
\hline & & Total & $42(100)$ \\
\hline \multirow{3}{*}{ Class II integron } & 1 & 2,200 & $2(50)$ \\
\hline & 2 & Without PCR products & $2(50)$ \\
\hline & & Total & $4(100)$ \\
\hline
\end{tabular}

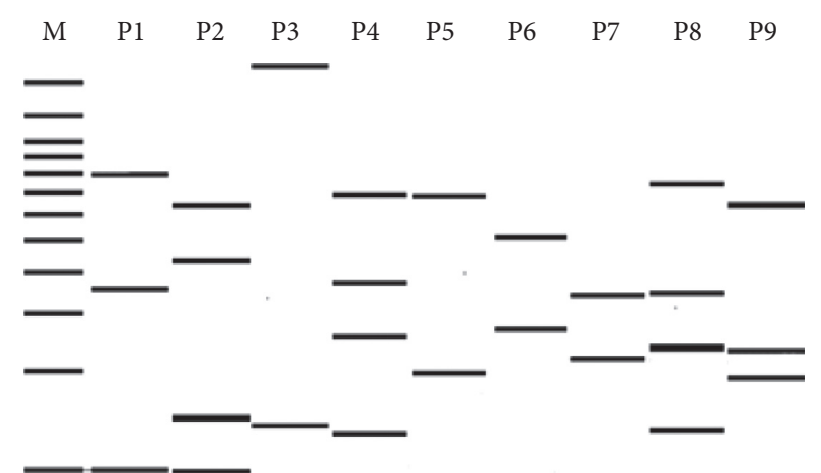

FIGURE 2: Schematic representation of the patterns obtained by digestion of integron I and II regions by HinfI restriction enzyme. M: $100 \mathrm{bp}$ DNA marker, P1: 2,200 bp, P2 and P3: 1,800 bp, P4: 1,600 bp, P5: 1,000 bp, P6: $750 \mathrm{bp}$ and $850 \mathrm{bp}, \mathrm{P} 7: 600 \mathrm{bp}$, and P8 and P9: 2,200 bp.

PMQR genes in Enterobacteriaceae [30]. Fluoroquinolone resistance and $E$. coli isolated from urine were found to have a significant correlation, where fluoroquinolones were one of the most important therapeutic regimens used in the treatment of uropathogens [31]. Therefore, this may explain the high prevalence of fluoroquinolone resistance among isolates from urinary tract infections, particularly in developing countries.

Integrons have been described as an acquired resistance mechanism by capturing, excising, and expressing gene cassettes through site-specific recombination, thus aiding in the spread of antibiotic resistance [32]. In this study, integrons were found in $44.77 \%$ of the tested isolates, which is similar to other studies $[10,12]$. Several studies, on the other hand, found a higher prevalence rate of integrons [33-35]. The present study revealed the predominance of integron I among quinolone-resistant isolates (59/80 isolates, $73.75 \%)$. In previous studies, integron I was more frequent among Gram-negative bacteria [36-38]. 
TABle 4: Size and number of gene cassettes amplified from integron-positive E. coli isolates' variable region.

\begin{tabular}{|c|c|c|}
\hline Gene cassette $(s)$ & Size (bp) & No. of isolates (\%) \\
\hline \multicolumn{3}{|c|}{ Class I integron } \\
\hline \multirow{4}{*}{$d f r B 4$} & 566 & $4(13.79)$ \\
\hline & 565 & $1(3.44)$ \\
\hline & 563 & $2(6.89)$ \\
\hline & 853 & $1(3.44)$ \\
\hline$d f r A 7$ & 775 & $1(3.44)$ \\
\hline \multirow[t]{5}{*}{ dfrA12 } & 1,828 & $1(3.44)$ \\
\hline & 362 & $1(3.44)$ \\
\hline & 785 & $1(3.44)$ \\
\hline & 1,615 & $1(3.44)$ \\
\hline & 1,618 & $1(3.44)$ \\
\hline \multirow[t]{6}{*}{ dfrA17 } & 1,619 & $1(3.44)$ \\
\hline & 1,624 & $1(3.44)$ \\
\hline & 1,625 & $1(3.44)$ \\
\hline & 1,642 & $1(3.44)$ \\
\hline & 1,675 & $1(3.44)$ \\
\hline & 1,027 & $1(3.44)$ \\
\hline \multirow{2}{*}{ aadA22 } & 1,032 & $1(3.44)$ \\
\hline & 1,622 & $1(3.44)$ \\
\hline \multirow{3}{*}{$d f r A 17-a a d A 5$} & 1,628 & $1(3.44)$ \\
\hline & 1,632 & $1(3.44)$ \\
\hline & 2,292 & $1(3.44)$ \\
\hline \multirow{2}{*}{ aadA2-hypothetical protein (orfF)-dfrA12 } & 1,863 & $1(3.44)$ \\
\hline & 2,142 & $1(3.44)$ \\
\hline \multicolumn{3}{|c|}{ Class II integron } \\
\hline$d f r A 17-a a d A 5$ & 2,169 & $1(3.44)$ \\
\hline \multirow{2}{*}{ dfrA1-sat2-aadA 1} & 2,172 & $1(3.44)$ \\
\hline & & 29 \\
\hline
\end{tabular}

TABLE 5: Characterization of 43 quinolone-resistant integron-positive clinical E. coli isolates.

\begin{tabular}{|c|c|c|c|c|c|c|c|c|c|}
\hline Isolate & Ward & Source & PMQR genes & $\begin{array}{c}\text { No. of } \\
\text { genes }\end{array}$ & Integrons & $\begin{array}{c}\text { Variable regions } \\
\text { size (bp) }\end{array}$ & $\begin{array}{c}\text { Gene cassette } \\
(\mathrm{s})\end{array}$ & $\begin{array}{c}\text { No. of gene } \\
\text { cassettes }\end{array}$ & $\begin{array}{c}\text { Accession } \\
\text { number }\end{array}$ \\
\hline 1 & UNC & Urine & $a a c\left(6^{\prime}\right)-I b$ & 3 & $\mathrm{I}$ & - & - & - & - \\
\hline 2 & $\mathrm{GEH}$ & Wound & $q n r S$ & 1 & $\mathrm{I}$ & - & - & - & - \\
\hline 3 & MDICU & Stool & $\begin{array}{c}q \eta r S, o q \times A, o q \times B \text {, and } \\
a a c\left(6^{\prime}\right)-I b\end{array}$ & 4 & $\begin{array}{c}\text { I } \\
\text { II }\end{array}$ & $\begin{array}{c}785 \\
- \\
\end{array}$ & $\begin{array}{c}d f r A 17 \\
-\end{array}$ & 1 & MW770328 \\
\hline 4 & $\mathrm{GEH}$ & Wound & $o q \times B$ and $a a c\left(6^{\prime}\right)-I b$ & 2 & $\mathrm{I}$ & 1,600 & $-{ }^{1}$ & - & - \\
\hline 10 & UNC & Urine & - & - & $\mathrm{I}$ & - & - & - & - \\
\hline 14 & GEH & Wound & $q n r S$ and $o q x B$ & 2 & $\mathrm{I}$ & 2,144 & $\begin{array}{c}\text { aadA2- } \\
\text { (orfF)-dfrA12 }\end{array}$ & 3 & MW770345 \\
\hline 15 & UNC & Urine & $\begin{array}{c}q n r A, q n r S, o q x B, \text { and } \\
a a c\left(6^{\prime}\right)-I b\end{array}$ & 4 & I & 1,632 & $\begin{array}{c}\text { dfrA17- } \\
\text { aadA5 }\end{array}$ & 2 & MW770333 \\
\hline 17 & UNC & Urine & $\begin{array}{c}q n r D, o q x B, q e p A \text {, and } \\
a a c\left(6^{\prime}\right)-I b\end{array}$ & 4 & I & 566 & $d f r B 4$ & 1 & MW770321 \\
\hline 20 & $\mathrm{GEH}$ & $\begin{array}{c}\text { Throat } \\
\text { swab }\end{array}$ & - & - & I & - & - & - & - \\
\hline 23 & $\mathrm{GEH}$ & Blood & $q n r A$ and $q n r S$ & 2 & $\mathrm{I}$ & 1,600 & $-{ }^{1}$ & - & - \\
\hline 24 & $\mathrm{GEH}$ & $\begin{array}{c}\text { Throat } \\
\text { swab }\end{array}$ & $q n r S$ & 1 & I & 1,826 & $\begin{array}{c}\text { aadA2- } \\
\text { (orfF)-dfrA12 }\end{array}$ & 3 & MW770343 \\
\hline 26 & MDICU & Urine & $\begin{array}{l}q n r D, o q x A, o q x B, \\
\text { and } a a c\left(6^{\prime}\right)-I b\end{array}$ & 4 & I & 1,628 & $\begin{array}{c}\text { aadA5- } \\
\text { dfrA17 } \\
\end{array}$ & 2 & MW770334 \\
\hline 30 & UNC & Urine & $q n r S$ and $o q x B$ & 2 & I & $\begin{array}{c}775 \\
1,027 \\
\end{array}$ & $\begin{array}{c}d f r A 7 \\
\text { aadA22 }\end{array}$ & $\begin{array}{l}1 \\
1\end{array}$ & $\begin{array}{l}\text { MW770329 } \\
\text { MW770331 }\end{array}$ \\
\hline 31 & UNC & Urine & $\begin{array}{c}q n r A, q n r B, q n r S, \\
o q x B, \text { and } a a c\left(6^{\prime}\right)-I b\end{array}$ & 5 & I & 1,615 & $d f r A 17$ & 1 & MW770335 \\
\hline 32 & UNC & Urine & $\begin{array}{c}o q x B, q e p A, \text { and } \\
\operatorname{aac}\left(6^{\prime}\right)-I b\end{array}$ & 3 & $\begin{array}{c}\text { I } \\
\text { III }\end{array}$ & $\begin{array}{c}2,292 \\
-\end{array}$ & $\begin{array}{c}d f r A 17- \\
\text { aadA5 } \\
-\end{array}$ & 2 & MW770346 \\
\hline
\end{tabular}


TABLE 5: Continued.

\begin{tabular}{|c|c|c|c|c|c|c|c|c|c|}
\hline Isolate & Ward & Source & PMQR genes & $\begin{array}{l}\text { No. of } \\
\text { genes }\end{array}$ & Integrons & $\begin{array}{l}\text { Variable regions } \\
\text { size }(\mathrm{bp})\end{array}$ & $\begin{array}{c}\text { Gene cassette } \\
\text { (s) }\end{array}$ & $\begin{array}{l}\text { No. of gene } \\
\text { cassettes }\end{array}$ & $\begin{array}{c}\text { Accession } \\
\text { number }\end{array}$ \\
\hline 33 & UNC & Urine & $\begin{array}{c}q n r B, q n r S, o q x B, \text { and } \\
q e p A, \operatorname{aac}\left(6^{\prime}\right)-I b\end{array}$ & 5 & $\begin{array}{c}\text { I } \\
\text { III }\end{array}$ & $\begin{array}{c}1,600 \\
-\end{array}$ & $\begin{array}{l}-1 \\
-\end{array}$ & - & - \\
\hline 38 & UNC & Urine & $\begin{array}{c}q n r B, q n r S, o q x B, \\
q e p A, \text { and } \operatorname{aac}\left(6^{\prime}\right)-I b\end{array}$ & 5 & $\mathrm{I}$ & 1,675 & $d f r A 17$ & 1 & MW770342 \\
\hline 40 & MDICU & Urine & $q n r S, o q x B$, and $q e p A$ & 3 & $\mathrm{I}$ & 1,600 & -1 & - & - \\
\hline 44 & UNC & Urine & - & - & $\mathrm{I}$ & - & - & - & - \\
\hline 45 & UNC & Urine & $q n r S$ and $a a c\left(6^{\prime}\right)-I b$ & 2 & I & $\begin{array}{c}566 \\
1,622\end{array}$ & $\begin{array}{c}d f r B 4 \\
\text { aadA5- } \\
d f r A 17\end{array}$ & 1 & $\begin{array}{l}\text { MW770322 } \\
\text { MW770336 }\end{array}$ \\
\hline 48 & MDICU & Urine & $a a c\left(6^{\prime}\right)-I b$ & 1 & $\mathrm{I}$ & 1,600 & -1 & - & - \\
\hline 51 & UNC & Urine & $\begin{array}{c}q n r D, q n r S, o q x A, \\
q e p A, \text { and } a a c\left(6^{\prime}\right)-I b\end{array}$ & 5 & $\begin{array}{l}\text { I } \\
\text { II }\end{array}$ & $\begin{array}{l}1,618 \\
2,172\end{array}$ & $\begin{array}{c}d f r A 17 \\
d f r A 1-s a t 2- \\
\text { aadA1 }\end{array}$ & $\begin{array}{l}1 \\
3\end{array}$ & $\begin{array}{l}\text { MW770337 } \\
\text { MW770347 }\end{array}$ \\
\hline 53 & UNC & Urine & $\begin{array}{c}q n r S, o q x A, q e p A, \text { and } \\
a a c\left(6^{\prime}\right)-I b\end{array}$ & 4 & $\begin{array}{l}\text { I } \\
\text { II }\end{array}$ & $\begin{array}{c}1,619 \\
-\end{array}$ & $\begin{array}{c}d f r A 17 \\
-\end{array}$ & 1 & MW770338 \\
\hline 54 & UNC & Urine & $a a c\left(6^{\prime}\right)-I b$ & 1 & $\begin{array}{c}\text { I } \\
\text { II }\end{array}$ & $-\overline{2,169}$ & $\begin{array}{c}- \\
d f r A 17- \\
\operatorname{aadA5}\end{array}$ & 2 & MW770348 \\
\hline 56 & UNC & Urine & $a a c\left(6^{\prime}\right)-I b$ & 1 & II & - & - & - & - \\
\hline 57 & UNC & Urine & - & - & $\mathrm{I}$ & - & - & - & - \\
\hline 63 & UNC & Urine & $a a c\left(6^{\prime}\right)-I b$ & 1 & $\mathrm{I}$ & 1,828 & $d f r A 12$ & 1 & MW770344 \\
\hline 64 & UNC & Urine & $q e p A$ & 1 & I & - & - & - & - \\
\hline 66 & UNC & Urine & $\begin{array}{c}o q x B, q e p A, \text { and } \\
a a c\left(6^{\prime}\right)-I b\end{array}$ & 3 & I & 1,032 & $\operatorname{aad} A 22$ & 1 & MW770332 \\
\hline 73 & GEH & Stool & $o q \times B$ & 1 & $\mathrm{I}$ & 1,600 & $-^{1}$ & - & - \\
\hline 74 & GEH & Stool & $\begin{array}{c}q n r S, o q x B, q e p A, \text { and } \\
\operatorname{aac}\left(6^{\prime}\right)-I b\end{array}$ & 4 & I & $\begin{array}{c}566 \\
1,624\end{array}$ & $\begin{array}{l}d f r B 4 \\
d f r A 17\end{array}$ & $\begin{array}{l}1 \\
1\end{array}$ & $\begin{array}{l}\text { MW770323 } \\
\text { MW770339 }\end{array}$ \\
\hline 84 & UNC & Urine & $\begin{array}{c}\text { oqxA, oqxB, qepA, and } \\
\operatorname{aac}\left(6^{\prime}\right)-I b\end{array}$ & 4 & I & - & - & - & - \\
\hline 85 & GEH & Stool & $\operatorname{aac}\left(6^{\prime}\right)-I b$ & 1 & $\mathrm{I}$ & 600 & -1 & - & - \\
\hline 87 & UNC & Urine & - & - & I & $\begin{array}{c}563 \\
856 \\
1,642 \\
\end{array}$ & $\begin{array}{c}d f r B 4 \\
d f r B 4 \\
d f r A 17\end{array}$ & $\begin{array}{l}1 \\
1 \\
1 \\
\end{array}$ & $\begin{array}{l}\text { MW770324 } \\
\text { MW770330 } \\
\text { MW770340 }\end{array}$ \\
\hline 89 & UNC & Urine & $q n r S, o q x B$, and $q e p A$ & 3 & $\mathrm{I}$ & 362 & dfrA17 & 1 & MW770320 \\
\hline 94 & UNC & Urine & - & - & $\mathrm{I}$ & - & - & - & - \\
\hline 95 & UNC & Urine & - & - & $\mathrm{I}$ & 1,600 & -1 & - & - \\
\hline 106 & UNC & Urine & $\begin{array}{c}q n r A, o q x A, o q x B, \text { and } \\
a a c\left(6^{\prime}\right)-I b\end{array}$ & 4 & I & 1,625 & $d f r A 17$ & 1 & MW770341 \\
\hline 107 & UNC & Urine & $o q \times B$ & 1 & $\mathrm{I}$ & 563 & $d f r B 4$ & 1 & MW770325 \\
\hline 110 & UNC & Urine & $o q \times B$ and $a a c\left(6^{\prime}\right)-I b$ & 2 & I & - & - & - & - \\
\hline 111 & UNC & Urine & $q e p A$ & 1 & $\mathrm{I}$ & 1,600 & -1 & - & - \\
\hline 116 & GEH & Stool & $\begin{array}{c}q n r S, q e p A, \text { and } \\
a a c\left(6^{\prime}\right)-I b\end{array}$ & 3 & $\mathrm{I}$ & 566 & $d f r B 4$ & 1 & MW770326 \\
\hline 129 & UNC & Urine & $q n r S$ & 1 & $\mathrm{I}$ & 565 & $d f r B 4$ & 1 & MW770327 \\
\hline
\end{tabular}

UNC: Urology and Nephrology Center, GEH: Gastroenterology Hospital, MDICU: Microbiology Diagnostic Infection Control Unit, and $-^{1}$ : not sequenced.

PMQR was detected in 36/43 (83.72\%) phenotypically fluoroquinolone-resistant isolates. Several studies showed similar results $[39,40]$. Other studies that investigated PMQR genes recorded variations in their prevalence rate even in the same country [41-44]. This variation may be due to the difference in study populations, types of isolates, selection criteria, and geographical distribution [45].
The active efflux pump genes, oq $x A B$ and qepA, had the highest prevalence $(72.22 \%)$, followed by $a a c\left(6^{\prime}\right)-\mathrm{Ib}-\mathrm{cr}$ gene (66.67\%) and $q n r$ genes (61.11\%). A study conducted in Egypt recorded $a a c\left(6^{\prime}\right)-I b-c r$ as the most frequent PMQR gene (61\%), followed by qnrS (43.3\%) [39]. Another study in China indicated a similar incidence rate of $a c c\left(6^{\prime}\right)-I b-c r$ and qepA genes [46]. Among the detected qnr genes, qnrS 
showed the highest prevalence, which agrees with previous studies [47-49]. Although detected in only $4.65 \%$ of our isolates, $q n r B$ gene was reported as one of the most abundant genes in other studies $[39,45,50]$. $q n r C$ gene was absent in all tested isolates, which agrees with other studies $[45,51,52]$. The qepA distribution rate in our results was similar to what was reported [52]. Several studies reported that $a a c\left(6^{\prime}\right)-I b-c r$ was detected more than $q n r$ genes in Enterobacteriaceae [39, 44, 53-55]. This may be attributed to the gene coding for this enzyme is highly prevalent in fluoroquinolone-resistant phenotypes. Besides, upon exposure to ciprofloxacin, this enzyme eases the selection of highresistant ciprofloxacin chromosomal mutants. In addition, the low level of fluoroquinolone resistance mediated by this enzyme can be converted to high-level resistance when coexisting with qnr proteins in the same bacterial isolates [56]. Seven isolates that showed quinolone resistance phenotypically did not harbor any of the tested genes on their plasmid. Their quinolone resistance may be attributed to other mechanisms that were not investigated in this study like chromosomal mutations of $\operatorname{gy} \mathrm{rA}$ or parC genes or due to impermeability of the membranes [39].

In the current study, the 36 isolates harboring the PMQR genes showed 23 different gene profiles. This result was consistent with what was reported earlier $[49,57]$. Concerning the coexistence of PMQR genes, no significant association was found. Similar results were reported previously $[49,58]$. Most isolates $(23 / 36$ isolates, $63.88 \%)$ showed more than 1 mechanism of PMQR, where $36.11 \%$ (13/36 isolates) of them had genes that encode the 3 PMQR mechanisms. A recent study in China has demonstrated the coexistence of three PMQR genes, including $a a c\left(6^{\prime}\right)-I b-c r, q n r S 2$, and oq $x A B$, on a multiple resistance plasmid [59]. A significant association was found between resistance mechanisms encoding efflux with $q n r$ and $a a c\left(6^{\prime}\right)-I b-c r$ genes $(P$-value $=0.0179$ and 0.0023 , respectively $)$. No significant association between resistance mechanisms encoded by $a a c\left(6^{\prime}\right)-I b-c r$ and $q n r$ genes was found, which contrasts with other studies [39, 60].

Among the 43 integron-positive E. coli isolates, gene cassettes were amplified in 32 isolates $(74.41 \%)$, which is comparable to the results reported by Zhang et al. (72.7\%) [61]. Although the isolates carried the integrase genes, the gene cassettes were not amplified in $26.19 \%$ and $50 \%$ of class I and II integron positive isolates, respectively. The lack or variation of a $3^{\prime}$-conserved segment [62] and the variation in the binding site of the primer or the extensive size of gene cassette $[63,64]$ may explain the absence of gene cassettes in these isolates. The most abundant variable regions were of size 600 and $1,600 \mathrm{bp}$ as they were found in 8 and 18 isolates, respectively. Similar results were reported earlier $[65,66]$. Identical restriction patterns of the same sized variable regions may be indicative of the presence of the same gene cassettes that were confirmed by sequencing.

Several gene cassettes belonging to class I integron have been described. Resistance to several antibiotics is mainly due to these cassettes [67]. In the current study, 23 out of 42 class I integron positive isolates carried 7 different gene cassettes (dfrA7, dfrA12, dfrA17, dfrB4, aadA22, dfrA17aadA5, and aadA2-orfF-dfrA12). The reported cassettes encode for resistance to trimethoprim and aminoglycosides and have been recorded among class I integrons in previous studies $[10,13,61,68,69]$. The diversity of class I integron cassettes is comparable to previous studies $[10,61]$ that reported 10 and 7 different gene cassettes among the tested isolates, respectively. In contrast to class I integrons, few different resistance cassettes have been associated with class II integrons [70]. In class II integron positive isolates of the current study, dfrA17-aadA5 and dfrA1-sat2-aadA1 gene cassettes were found. Zhang et al. [61] reported dfrA1-sat2aadA1 as a single gene cassette among class II integron positive isolates.

The gene cassettes in the present study showed the dominance of $d f r$ genes within class I and II integrons. Similar results were reported previously $[10,34,69]$. This may be explained by the extensive use of trimethoprim as a first-line treatment for urinary tract infections in both hospital and community settings [34]. $d f r A 17, d f r B 4$, and $d f r A 17-a a d A 5$ gene cassettes were found in nine, eight, and five isolates, respectively. This may be due to the interspecies transfer of the integrons. Integrons disseminate in hospitals through the cross-transmission of integron-carrying clones from one patient to another [34, 71].

Concerning the variable region of $1,600 \mathrm{bp}$, four and seven variable regions carried $d f r A 17$-aadA5 and $d f r A 17$ gene cassettes, respectively. It is worth mentioning that these seven variable regions had also the aadA5 gene, which had a frameshift mutation that resulted in several internal stop codons and gaps. Similar results were reported by Chen et al. [66] where the $1,600 \mathrm{bp}$ amplicon harbored $d f r A 17$-aadA5 genes. This cassette array was reported in other bacteria and other regions around the world $[9,72-75]$. This array can spread all over the world by self-transferable plasmids in humans or animals [76].

No PMQR genes were detected in the amplified gene cassettes. This indicates that quinolone resistance genes are not cassette-born. This coincides with the study of Kubomura et al. who found that except for $d f r A$ and aadA genes, the antibiotic resistance genes are mostly found outside the integrons [69]. This may also suggest that resistance is related to other mobile genetic elements such as plasmids and transposons [77].

\section{Conclusion}

This study indicates the high prevalence of quinolone resistance and integrons in E. coli isolates, especially those from urine. PMQR genes were widely distributed among the tested isolates, implying lateral gene transfer. Gene sequencing of the amplified variable regions of integrons revealed that the most predominant gene cassettes were for trimethoprim and aminoglycoside resistance. Although PMQR genes are not cassette-born, they were associated with integrons' presence on the plasmids. Future studies to explain this association phenomenon should be performed. Continued surveillance of PMQR and integrons should be 
conducted to control their spread and the associated health risks.

\section{Data Availability}

The data used to support the findings of this study are available from the corresponding author upon request.

\section{Conflicts of Interest}

The authors declare that they have no conflict of interest.

\section{Authors' Contributions}

The authors contributed equally to this work. All the authors contributed to the conception of the study, design of the work, analysis, and drafting the work and read and approved the final manuscript.

\section{References}

[1] O. Tenaillon, D. Skurnik, B. Picard, and E. Denamur, "The population genetics of commensal Escherichia coli," Nature Reviews Microbiology, vol. 8, no. 3, pp. 207-217, 2010.

[2] N. S. Alharbi, J. M. Khaled, S. Kadaikunnan et al., "Prevalence of Escherichia coli strains resistance to antibiotics in wound infections and raw milk," Saudi Journal of Biological Sciences, vol. 26, no. 7, pp. 1557-1562, 2019.

[3] D. C. Hooper and G. A. Jacoby, "Mechanisms of drug resistance: quinolone resistance," Annals of the New York Academy of Sciences, vol. 1354, no. 1, pp. 12-31, 2015.

[4] J. M. Rodríguez-Martínez, J. Machuca, M. E. Cano, J. Calvo, L. Martínez-Martínez, and A. Pascual, "Plasmid-mediated quinolone resistance: two decades on," Drug Resistance Updates, vol. 29, pp. 13-29, 2016.

[5] S. Cocchi, E. Grasselli, M. Gutacker, C. Benagli, M. Convert, and J.-C. Piffaretti, "Distribution and characterization of integrons in Escherichia coli strains of animal and human origin," FEMS Immunology \& Medical Microbiology, vol. 50, no. 1, pp. 126-132, 2007.

[6] J. Cury, T. Jové, M. Touchon, B. Néron, and E. P. Rocha, "Identification and analysis of integrons and cassette arrays in bacterial genomes," Nucleic Acids Research, vol. 44, no. 10, pp. 4539-4550, 2016.

[7] X. Yang, W. Zou, J. Zeng et al., "Prevalence of antimicrobial resistance and integron gene cassettes in Escherichia coli isolated from yaks (Poephagus grunniens) in Aba Tibetan Autonomous Prefecture, China," Microbial Pathogenesis, vol. 111, pp. 274-279, 2017.

[8] J. Huang, F. Lan, Y. Lu, and B. Li, "Characterization of integrons and antimicrobial resistance in Escherichia coli sequence type 131 isolates," The Canadian Journal of Infectious Diseases \& Medical Microbiology, vol. 2020, Article ID 3826186, 8 pages, 2020.

[9] H. Y. Kang, Y. S. Jeong, J. Y. Oh et al., "Characterization of antimicrobial resistance and class 1 integrons found in Escherichia coli isolates from humans and animals in Korea," Journal of Antimicrobial Chemotherapy, vol. 55, no. 5, pp. 639-644, 2005.

[10] B. W. Odetoyin, A. S. Labar, A. Lamikanra, A. O. Aboderin, and I. N. Okeke, "Classes 1 and 2 integrons in faecal Escherichia coli strains isolated from mother-child pairs in Nigeria," PLoS One, vol. 12, no. 8, Article ID e0183383, 2017.
[11] A. Tomova, L. Ivanova, A. H. Buschmann, H. P. Godfrey, and F. C. Cabello, "Plasmid-mediated quinolone resistance (PMQR) genes and class 1 integrons in quinolone-resistant marine bacteria and clinical isolates of Escherichia coli from an aquacultural area," Microbial Ecology, vol. 75, no. 1, pp. 104-112, 2018.

[12] M. Amin, S. Dibachi, and M. Shahin, "Prevalence of class 1 integrons and plasmid-mediated qnr-genes among Enterobacter isolates obtained from hospitalized patients in Ahvaz, Iran," Infezioni in Medicina, Le, vol. 25, no. 4, pp. 351-357, 2017.

[13] K. W. Seo and Y. J. Lee, "Prevalence and characterization of plasmid mediated quinolone resistance genes and class 1 integrons among multidrug-resistant Escherichia coli isolates from chicken meat," The Journal of Applied Poultry Research, vol. 28, no. 3, pp. 761-770, 2019.

[14] D. H. Bergey, N. R. Krieg, and J. G. Holt, Bergey's Manual of Systemic Bacteriology, William and Wilkins, Baltimore, MD, USA, 1984.

[15] Clinical and Laboratory Standard Institute, CLSI; M100-S24 Performance Standards For Antimicrobial Susceptibility Testing. Twenty-four Informational Supplements, CLSI, Wayne, PA, USA, 2014.

[16] M. D. Englen and L. C. Kelley, "A rapid DNA isolation procedure for the identification of Campylobacter jejuni by the polymerase chain reaction," Letters in Applied Microbiology, vol. 31, no. 6, pp. 421-426, 2000.

[17] A. FarajzadehSheikh, H. Veisi, M. Shahin, M. Getso, and A. Farahani, "Frequency of quinolone resistance genes among extended-spectrum $\beta$-lactamase (ESBL)-producing Escherichia coli strains isolated from urinary tract infections," Tropical Medicine and Health, vol. 47, no. 1, p. 19, 2019.

[18] S. SAHIN, "Determination of the ciprofloxacin-resistant Escherichia coli isolated from chicken meat in Turkey," Journal of the Hellenic Veterinary Medical Society, vol. 71, no. 3, pp. 2291-2300, 2020.

[19] M. Y. Yoon, Y. B. Kim, J. S. Ha et al., "Molecular characteristics of fluoroquinolone-resistant avian pathogenic Escherichia coli isolated from broiler chickens," Poultry Science, vol. 99, no. 7, pp. 3628-3636, 2020.

[20] M. Goudarzi, M. Azad, and S. S. Seyedjavadi, "Prevalence of plasmid-mediated quinolone resistance determinants and OqxAB efflux pumps among extended-spectrum $\beta$-lactamase producing Klebsiella pneumoniae isolated from patients with nosocomial urinary tract infection in Tehran, Iran," Scientific, vol. 2015, Article ID 518167, 7 pages, 2015.

[21] D. E. Rizk and A. M. El-Mahdy, "Emergence of class 1 to 3 integrons among members of Enterobacteriaceae in Egypt," Microbial Pathogenesis, vol. 112, pp. 50-56, 2017.

[22] A. Osińska, M. Harnisz, and E. Korzeniewska, "Prevalence of plasmid-mediated multidrug resistance determinants in fluoroquinolone-resistant bacteria isolated from sewage and surface water," Environmental Science and Pollution Research, vol. 23, no. 11, pp. 10818-10831, 2016.

[23] S. A. Taha, H. H. Omar, and wH. Hassan, "Characterization of plasmid-mediated qnrA and qnrB genes among Enterobacteriaceae strains: quinolone resistance and ESBL production in Ismailia, Egypt," Egyptian Journal of Medical Human Genetics, vol. 20, no. 26, 2019.

[24] G. V. Sanchez, S. J. E. Adams, A. M. G. Baird, R. N. Master, R. B. Clark, and J. M. Bordon, "Escherichia coli antimicrobial resistance increased faster among geriatric outpatients compared with adult outpatients in the USA, 2000-10," 
Journal of Antimicrobial Chemotherapy, vol. 68, no. 8, pp. 1838-1841, 2013.

[25] B. Yang, F. Yang, S. Wang et al., "Analysis of the spectrum and antibiotic resistance of uropathogens in outpatients at a tertiary hospital," Journal of Chemotherapy, vol. 30, no. 3, pp. 145-149, 2018.

[26] M. Seitz, C. Stief, and R. Waidelich, "Local epidemiology and resistance profiles in acute uncomplicated cystitis (AUC) in women: a prospective cohort study in an urban urological ambulatory setting," BMC Infectious Diseases, vol. 17, no. 1, p. $685,2017$.

[27] H. Hayami, S. Takahashi, K. Ishikawa et al., "Nationwide surveillance of bacterial pathogens from patients with acute uncomplicated cystitis conducted by the Japanese surveillance committee during 2009 and 2010: antimicrobial susceptibility of Escherichia coli and Staphylococcus saprophyticus," Journal of Infection and Chemotherapy: Official Journal of the Japan Society of Chemotherapy, vol. 19, no. 3, pp. 393-403, 2013.

[28] Australian Commission on Safety and Quality in Health Care, Preliminary Report on Antimicrobial Use and Resistance in Australia (AURA), ACSQHC, Sydney, Australia, 2014.

[29] D. S. Lee, S.-J. Lee, and H.-S. Choe, "Community-acquired urinary tract infection by Escherichia coli in the era of antibiotic resistance," BioMed Research International, vol. 2018, Article ID 7656752, 14 pages, 2018.

[30] J. Strahilevitz, G. A. Jacoby, D. C. Hooper, and A. Robicsek, "Plasmid-mediated quinolone resistance: a multifaceted threat," Clinical Microbiology Reviews, vol. 22, no. 4, pp. 664-689, 2009.

[31] T. Gururaju, T. Kasturi, and C. Mallikarjuna reddy, “A study of antibiotic sensitivity pattern and detection of fluoroquinolones resistance to Escherichia coli from urinary tract infections," International Journal of Current Microbiology and Applied Sciences, vol. 4, no. 2, pp. 799-805, 2015.

[32] M. Karimi Dehkordi, M. Halaji, and S. Nouri, "Prevalence of class 1 integron in Escherichia coli isolated from animal sources in Iran: a systematic review and meta-analysis," Tropical Medicine and Health, vol. 48, no. 1, p. 16, 2020.

[33] B. F. Araújo, P. A. D. Campos, S. Royer et al., "High frequency of the combined presence of QRDR mutations and PMQR determinants in multidrug-resistant Klebsiella pneumoniae and Escherichia coli isolates from nosocomial and community-acquired infections," Journal of Medical Microbiology, vol. 66, no. 8, pp. 1144-1150, 2017.

[34] M. M. Malek, F. A. Amer, A. A. Allam, R. H. El-Sokkary, T. Gheith, and M. A. Arafa, "Occurrence of classes I and II integrons in Enterobacteriaceae collected from Zagazig University Hospitals, Egypt," Frontiers in Microbiology, vol. 6, p. 601, 2015.

[35] N. S. Singh, N. Singhal, M. Kumar, and J. S. Virdi, "High prevalence of drug resistance and class 1 integrons in Escherichia coli isolated from river Yamuna, India: a serious public health risk," Frontiers in Microbiology, vol. 12, Article ID 621564, 2021.

[36] Y. Deng, X. Bao, L. Ji et al., "Resistance integrons: class 1, 2 and 3 integrons," Annals of Clinical Microbiology and Antimicrobials, vol. 14, no. 1, p. 45, 2015.

[37] F. Zeeshan Khan, T. Nawaz, Z. A. Mirani, S. Khan, Y. Raza, and S. U. Kazmi, "Study of class 1 integrons in multidrugresistant uropathogenic Escherichia coli isolated from different hospitals in Karachi," Iranian Journal of Basic Medical Sciences, vol. 21, no. 10, pp. 1079-1082, 2018.

[38] D. Jones-Dias, V. Manageiro, E. Ferreira et al., "Architecture of class 1,2 , and 3 integrons from gram negative bacteria recovered among fruits and vegetables," Frontiers in Microbiology, vol. 7, p. 1400, 2016.

[39] N. E. Esmaeel, M. A. Gerges, T. A. Hosny, A. R. Ali, and M. G. Gebriel, "Detection of chromosomal and plasmidmediated quinolone resistance among Escherichia coli isolated from urinary tract infection cases; Zagazig university hospitals, Egypt," Infection and Drug Resistance, vol. 13, pp. 413421, 2020.

[40] M. Khalil, R. Elsherif, D. Ghaith et al., "Quinolone resistance detection by PCR-RFLP and multiplex-PCR among extendedspectrum $\beta$ - lactamase producing Enterobacteriaceae," International Journal of Clinical \& Medical Microbiology, vol. 2, no. 1, p. 119, 2017.

[41] H. H. Al-Hasnawy, M. R. Jodi, and H. J. Hamza, "Molecular characterization and sequence analysis of plasmid-mediated quinolone resistance genes in extended-spectrum beta-lactamases producing uropathogenic Escherichia coli in Babylon Province, Iraq," Reviews in Medical Microbiology, vol. 29, no. 3, pp. 129-135, 2018.

[42] Y. Malekzadegan, E. Rastegar, M. Moradi, H. Heidari, and H. Sedigh Ebrahim-Saraie, "Prevalence of quinolone-resistant uropathogenic Escherichia coli in a tertiary care hospital in south Iran," Infection and Drug Resistance, vol. 12, pp. 1683-1689, 2019.

[43] M. H. Al-Agamy, A. Aljallal, H. H. Radwan, and A. M. Shibl, "Characterization of carbapenemases, ESBLs, and plasmidmediated quinolone determinants in carbapenem-insensitive Escherichia coli and Klebsiella pneumoniae in Riyadh hospitals," Journal of Infection and Public Health, vol. 11, no. 1, pp. 64-68, 2018.

[44] C.-Y. Kao, H.-M. Wu, W.-H. Lin et al., "Plasmid-mediated quinolone resistance determinants in quinolone-resistant Escherichia coli isolated from patients with bacteremia in a university hospital in Taiwan, 2001-2015," Scientific Reports, vol. 6, no. 1, p. 32281, 2016.

[45] D. N. Kotb, W. K. Mahdy, M. S. Mahmoud, and R. M. M. Khairy, "Impact of co-existence of PMQR genes and QRDR mutations on fluoroquinolones resistance in Enterobacteriaceae strains isolated from community and hospital acquired UTIs," BMC Infectious Diseases, vol. 19, no. 1, p. 979, 2019.

[46] J. Yang, Y. Luo, S. Cui, W. Wang, and L. Han, "Diverse phenotypic and genotypic characterization among Clinical Klebsiella pneumoniae and Escherichia coli isolates carrying plasmid-mediated quinolone resistance determinants," $M i$ crobial Drug Resistance, vol. 17, no. 3, pp. 363-367, 2011.

[47] S. M. Hamed, K. M. A. Aboshanab, H. A. El-Mahallawy, M. M. Helmy, M. S. Ashour, and W. F. Elkhatib, "Plasmidmediated quinolone resistance in gram-negative pathogens isolated from cancer patients in Egypt," Microbial Drug Resistance, vol. 24, no. 9, pp. 1316-1325, 2018.

[48] H. O. Khalifa, A. M. Soliman, A. M. Ahmed et al., "High prevalence of antimicrobial resistance in gram-negative bacteria isolated from clinical settings in Egypt: recalling for judicious use of conventional antimicrobials in developing nations," Microbial Drug Resistance, vol. 25, no. 3, pp. 371-385, 2019.

[49] B. Li, Y. Chen, Z. Wu, Z. Zhao, J. Wu, and Y. Cao, "Prevalence of plasmid-mediated quinolone resistance genes among Escherichia coli in the gut of healthy people in Fuzhou, China," Annals of Laboratory Medicine, vol. 38, no. 4, pp. 384-386, 2018.

[50] M. F. El-Badawy, W. M. Tawakol, S. W. El-Far et al., "Molecular identification of aminoglycoside-modifying enzymes and plasmid-mediated quinolone resistance genes among 
Klebsiella pneumoniae clinical isolates recovered from Egyptian patients," International Journal of Microbiology, vol. 2017, Article ID 8050432, 12 pages, 2017.

[51] O. Szabó, D. Gulyás, N. Szabó, K. Kristóf, B. Kocsis, and D. Szabó, "Plasmid-mediated quinolone resistance determinants in Enterobacteriaceae from urine clinical samples," Acta Microbiologica et Immunologica Hungarica, vol. 65, no. 3, pp. 255-265, 2018.

[52] M. E. S. Zaki, M. A. El Salam, and O. A. Faried, "Study of plasmid-mediated quinolone resistance in Escherichia coli from nosocomial urinary tract infections," Infectious Disorders-Drug Targets, vol. 21, no. 2, pp. 243-247, 2021.

[53] R. Azargun, M. R. Sadeghi, M. H. Soroush Barhaghi et al., "The prevalence of plasmid-mediated quinolone resistance and ESBL-production in Enterobacteriaceae isolated from urinary tract infections," Infection and Drug Resistance, vol. 11, pp. 1007-1014, 2018.

[54] A. Majlesi, R. K. Kakhki, A. S. Mozaffari Nejad et al., "Detection of plasmid-mediated quinolone resistance in clinical isolates of Enterobacteriaceae strains in Hamadan, West of Iran," Saudi Journal of Biological Sciences, vol. 25, no. 3, pp. 426-430, 2018.

[55] A. Akya, R. Chegene Lorestani, A. Elahi, and K. Ghadiri, “The impact of mutations in topoisomerase genes and the plasmidmediated quinolone resistance (PMQR) determinants on the resistance to fluoroquinolones in Klebsiella pneumoniae," Archives of Clinical Infectious Diseases, vol. 12, no. 4, Article ID e57290, 2017.

[56] A. Robicsek, J. Strahilevitz, G. A. Jacoby et al., "Fluoroquinolone-modifying enzyme: a new adaptation of a common aminoglycoside acetyltransferase," Nature Medicine, vol. 12, no. 1, pp. 83-88, 2006.

[57] H. Y. Yang, Y. S. Nam, and H. J. Lee, "Prevalence of plasmidmediated quinolone resistance genes among ciprofloxacinnonsusceptible Escherichia coli and Klebsiella pneumoniae isolated from blood cultures in Korea," The Canadian Journal of Infectious Diseases \& Medical Microbiology, vol. 25, no. 3, pp. 163-169, 2014.

[58] Y. Malekzadegan, E. Rastegar, M. Moradi, H. Heidari, and H. Sedigh Ebrahim-Saraie, "Prevalence of quinolone-resistant uropathogenic Escherichia coli in a tertiary care hospital in south Iran [Response to letter]," Infection and Drug Resistance, vol. 12, pp. 2175-2176, 2019.

[59] Y. Tao, K. Zhou, L. Xie et al., "Emerging coexistence of three PMQR genes on a multiple resistance plasmid with a new surrounding genetic structure of qnrS2 in E. coli in China," Antimicrobial Resistance \& Infection Control, vol. 9, no. 1, p. 52, 2020.

[60] G. A. Jacoby, J. Strahilevitz, and D. C. Hooper, "Plasmidmediated quinolone resistance," Microbiology Spectrum, vol. 2, no. 5, 2014.

[61] S. Zhang, H. Yang, M. U. Rehman et al., "Class 1 integrons as predominant carriers in Escherichia coli isolates from waterfowls in Hainan, China," Ecotoxicology and Environmental Safety, vol. 183, Article ID 109514, 2019.

[62] A. Moura, I. Henriques, R. Ribeiro, and A. Correia, "Prevalence and characterization of integrons from bacteria isolated from a slaughterhouse wastewater treatment plant," Journal of Antimicrobial Chemotherapy, vol. 60, no. 6, pp. 1243-1250, 2007.

[63] F. E. Dawes, A. Kuzevski, K. A. Bettelheim, M. A. Hornitzky, S. P. Djordjevic, and M. J. Walker, "Distribution of class 1 integrons with IS26-mediated deletions in their $3^{\prime}$-conserved segments in Escherichia coli of human and animal origin," PLoS One, vol. 5, no. 9, Article ID e12754, 2010.
[64] A. Japoni-Nejad, S. Farshad, A. van Belkum, and E. GhaznaviRad, "Novel cassette array in a class 1 integron in clinical isolates of Acinetobacter baumannii from central Iran," International Journal of Medical Microbiology, vol. 303, no. 8, pp. 645-650, 2013.

[65] M. Kargar, Z. Mohammadalipour, A. Doosti, S. Lorzadeh, and A. Japoni-Nejad, "High prevalence of class 1 to 3 integrons among multidrug-resistant diarrheagenic Escherichia coli in southwest of Iran," Osong Public Health and Research Perspectives, vol. 5, no. 4, pp. 193-198, 2014.

[66] J. Chen, Z. Su, Y. Liu et al., "Identification and characterization of class 1 integrons among Pseudomonas aeruginosa isolates from patients in Zhenjiang, China," International Journal of Infectious Diseases, vol. 13, no. 6, pp. 717-721, 2009.

[67] M. Kaushik, S. Kumar, R. K. Kapoor, J. S. Virdi, and P. Gulati, "Integrons in Enterobacteriaceae: diversity, distribution and epidemiology," International Journal of Antimicrobial Agents, vol. 51, no. 2, pp. 167-176, 2018.

[68] H.-C. Su, G.-G. Ying, R. Tao, R.-Q. Zhang, J.-L. Zhao, and Y.-S. Liu, "Class 1 and 2 integrons, sul resistance genes and antibiotic resistance in Escherichia coli isolated from Dongjiang River, South China," Environmental Pollution, vol. 169, pp. 42-49, 2012.

[69] A. Kubomura, T. Sekizuka, D. Onozuka et al., "Truncated class 1 integron gene cassette arrays contribute to antimicrobial resistance of diarrheagenic Escherichia coli," BioMed Research International, vol. 2020, Article ID 4908189, 9 pages, 2020.

[70] D. Mazel, "Integrons: agents of bacterial evolution," Nature Reviews Microbiology, vol. 4, no. 8, pp. 608-620, 2006.

[71] M. A. Leverstein-van Hall, A. T. A. Box, H. E. M. Blok, A. Paauw, A. C. Fluit, and J. Verhoef, "Evidence of extensive interspecies transfer of integron-mediated antimicrobial resistance genes among multidrug-resistant Enterobacteriaceae in a clinical setting," The Journal of Infectious Diseases, vol. 186, no. 1, pp. 49-56, 2002.

[72] H. Zhang, L. Shi, L. Li et al., "Identification and characterization of class 1 integron resistance gene cassettes among Salmonella strains isolated from healthy humans in China," Microbiology and Immunology, vol. 48, no. 9, pp. 639-645, 2004, Epub 2004/09/24.

[73] L.-L. Chang, T.-M. Chang, and C.-Y. Chang, "Variable gene cassette patterns of class 1 integron-associated drug-resistant Escherichia coli in Taiwan," The Kaohsiung Journal of Medical Sciences, vol. 23, no. 6, pp. 273-280, 2007.

[74] P. A. White, C. J. McIver, Y.-M. Deng, and W. D. Rawlinson, "Characterisation of two new gene cassettes, aadA5 and dfrA17,” FEMS Microbiology Letters, vol. 182, no. 2, pp. 265-269, 2000.

[75] B.-A. Lindstedt, E. Heir, I. Nygard, and G. Kapperud, "Characterization of class I integrons in clinical strains of Salmonella enterica subsp. enterica serovars Typhimurium and Enteritidis from Norwegian hospitals," Journal of Medical Microbiology, vol. 52, no. 2, pp. 141-149, 2003.

[76] B. Guerra, E. Junker, A. Schroeter, B. Malorny, S. Lehmann, and R. Helmuth, "Phenotypic and genotypic characterization of antimicrobial resistance in German Escherichia coli isolates from cattle, swine and poultry," Journal of Antimicrobial Chemotherapy, vol. 52, no. 3, pp. 489-492, 2003.

[77] N. Canal, K. L. Meneghetti, C. P. de Almeida, M. da Rosa Bastos, L. M. Otton, and G. Corção, "Characterization of the variable region in the class 1 integron of antimicrobial-resistant Escherichia coli isolated from surface water," Brazilian Journal of Microbiology, vol. 47, no. 2, pp. 337-344, 2016. 